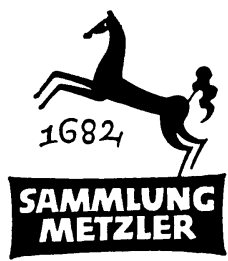

REALIEN ZUR LITERATUR

ABT. D

LITERATURGESCHICHTE 
HELENE M. KASTINGER RILEY

\title{
Clemens Brentano
}

\author{
MCMLXXXV \\ J.B. METZLERSCHE VERLAGSBUCHHANDLUNG \\ STUTTGART
}


CIP-Kurztitelaufnahme der Deutschen Bibliothek

Riley, Helene M. Kastinger:

Clemens Brentano / Helene M. Kastinger Riley. -

Stuttgart : Metzler, 1985.

ISBN 978-3-476-10213-3

(Sammlung Metzler ; M 213 : Abt. D, Literaturgeschichte)

NE: GT

ISBN 978-3-476-10213-3

ISBN 978-3-476-03917-0 (eBook)

DOI 10.1007/978-3-476-03917-0

M 213

(C) 1985 Springer-Verlag GmbH Deutschland

Ursprünglich erschienen bei J. B. Metzlersche Verlagsbuchhandlung und Carl Ernst Poeschel Verlag GmbH in Stuttgart 1985 
Verzeichnis der Abkürzungen . . . . . . . . VIII

I. Materialien ................ 1

1. Hilfsmittel der Forschung . . . . . . . . . 1

2. Nachlaß und Nachlaßeditionen $\ldots \ldots \ldots \ldots$

3. Die wichtigsten Ausgaben . . . . . . . . . 5

4. Briefe ............... 8

II. Beziehungen und Wirkungen . . . . . . . . 12

1. Kindheit und Jugend (1778-1797) . . . . . . 12

2. Die romantische Schulung: Jena, Göttingen, Heidelberg (1798-1808) . . . . . 18

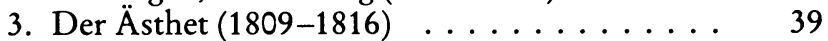

4. Luise Hensel und A. K. Emmerick (1817-1823) 50

5. Die Reisejahre (1824-1833) . . . . . . . 62

6. München $(1834-1842) \ldots \ldots \ldots \ldots$

III. Das Werk . . . . . . . . . . . 76

1. Lyrik

a. Problemstellungen der Forschung . . . . 76

b. Kommentar zu einzelnen Werken ..... 81

2. Prosa

a. Forschungsüberblick . . . . . . . . 93

b. Kommentar zu einzelnen Werken ..... 97

3. Märchen

a. Problemstellungen der Forschung . . . . 108

b. Kommentar zu einzelnen Werken ..... 110

4. Bühnenwerke

a. Forschungsüberblick . . . . . . . . . 125

b. Kommentar zu einzelnen Werken ..... 129

5. Religiöse Schriften . . . . . . . . . . . . . 141

6. Kritische Schriften . . . . . . . . . . . . . . 145

7. Briefe ................... 154

Personenregister ................. 156

Ort-und Sachregister . . . . . . . . . . . . 162 
Der vorliegende Band soll eine erste Einführung für den an Brentano Interessierten bieten, zugleich aber auch dem Brentano-Forscher als zusammenfassendes Hilfsmittel dienen. Da sich die gegenwärtige Forschungslage in einem Übergang befindet, der durch die seit langem geforderte und nun erfolgende Publikation eine historisch-kritischen Ausgabe (HKA) verursacht ist, muß die Darstellung zwangsläufig transitorisch bleiben. Sie ist Manifestation einer am Scheidewege angelangten Kritik und kann höchstens richtungsweisend wirken, ohne auf Vollkommenheit Anspruch zu erheben. Während der Erarbeitung der einzelnen Bände der HKA werden nicht nur neue Funde und Erkenntnisse von der Mitarbeitern publiziert, auch das gesamte Brentano-Material in der umfangreichen Sammlung des FDH und beisteuernder Archive ist dem Nichtbeteiligten unzugänglich. Die B-Forschung steht am Anfang einer neuen Epoche, die eine Neuorientierung und Neubestimmung von Leben und Werk des Dichters erwarten läßt.

Der Perspektive dieses Bandes liegt die Prämisse zugrunde, daß Brentanos Arbeits- und Lebensgemeinschaften sowohl auf seine Themenwahl wie auf seine literarische Produktion großen Einfluß ausübten. Die mit seiner Schaffensdynamik eng verflochtenen Beziehungen zur zeitgenössischen Literatur- und Gesellschaftsgeschichte bilden mit ihren wichtigsten Charakterzügen einen wesentlichen Teil der Erörterungen. Die restlichen Abschnitte sind der kritischen und bibliographischen Erfassung des Werks gewidmet, wobei ein Hauptaugenmerk auf einzelne Problemkreise, bzw. auf das noch zu Erarbeitende in der Forschung gerichtet wird.

Die Literaturangaben verzeichnen die wichtigsten Arbeiten neuerer Zeit, wobei nach Möglichkeit das bis 1983 Erschienene berücksichtigt wurde. Schon aus Platzgründen ist es unmöglich, ein Gesamtverzeichnis der B-Literatur zu liefern. Da zwischen 1970 und 1978 drei ausgezeichnete Forschungsberichte erschienen sind (Fetzer, Frühwald, Gajek) und für die ältere Forschung ebenfalls in Zeitabständen Zusammenfassungen der Literatur vorliegen (Mallons Bibliographie, u. a.) wird auf diese Arbeiten verwiesen. Schließlich sind alle jene Werke von der Diskussion und der bibliographischen Erfassung ausgeschlossen worden, die nicht eindeutig von Brentano stammen. Hierzu gehören die ihm manchmal zugeschriebenen "Nachtwachen" 
des Bonaventura, die Übersetzungen und mehrere kleinere Schriften.

Zu besonderem Dank bin ich meinen verehrten Kollegen, den Herren Professoren Dr. Fetzer, Frühwald und Gajek verpflichtet, die die Güte hatten, das Manuskript durchzusehen und mit wertvollen Hinweisen zu bereichern.

Helene M. Kastinger Riley New Haven, Ct., März 1984 
AK

$\mathrm{B}, \mathrm{CB}$

$\mathrm{BdK}$

Beutler

$\mathrm{BM}$

CB I

$\mathrm{CB} / \mathrm{BG}$

CBGS

CG

DVjS

EG

Eichendorff 4

ELN

FB

$\mathrm{FDH}$

Feilchenfeldt

$\mathrm{Fr}$

GB, GB 2

GGB

GLL

GR

GRM

GQ

HKA

Homo poeta

$\mathrm{Jb}, \mathrm{Jbb}$

JbFDH

JbDSG
Ausstellungskatalog: CB 1778-1842. Ausstellung FDH, Frankfurter Goethe-Museum. 5. Sept. bis 31. Dez. 1978, hrg. v. D. Lüders (Frankfurt, 1978).

Brentano, Clemens Brentano

CB. Beiträge des Kolloquiums im FDH 1978, hrg. v. D. Lüders (Tübingen, 1980)

Ernst Beutler: "Briefe aus dem B-Kreis «, JbFDH (1934/35), 367-455

Briefwechsel zwischen CB und Sophie Mereau, hrg. v. H. Amelung, 2 Bde (Leipzig, 1. und 2. Aufl., 1908)

CB. Werke in zwei Bänden, hrg. von v. F. Kemp/ W. Frühwald, Bd. 1 (München, 1972)

$C B$ und die Brüder Grimm, von R. Steig (Stuttgart, 1914)

CB's Gesammelte Schriften, hrg. v. Sauerländer Verlag (Frankfurt, 1852-1855)

Colloquia Germanica

Deutsche Vierteljahrsschrift für Literaturwissenschaft und Geistesgeschichte

Etudes Germaniques

J. v. Eichendorff. Werke, hrg. v. R. Strasser, Bd. 4 (Zürich, 1965)

English Language Notes

Forschungsbericht

Freies Deutsches Hochstift

CB/Philipp Otto Runge. Briefwechsel, hrg. v. K. Feilchenfeldt (Frankfurt, 1974)

CBs Frühlingskranz aus Jugendbriefen ihm geflochten, hrg. v. Bettina v. Arnim (Leipzig, 1909)

CB, Gesammelte Briefe, Bd. 1-2 (= CB. Gesammelte Schriften, Bd. 8-9. Frankfurt, 1855)

J. v. Görres. Gesammelte Briefe, 3 Bde., hrg. v. M. Görres/F. Binder (München, 1858-1874)

German Life and Letters

Germanic Rewiev

Germanisch-Romanische Monatsschrift

German Quarterly

$\mathrm{CB}$. Sämtliche Werke und Briefe, historisch-kritische Ausgabe, hrg. v. J. Behrens, W. Frühwald, D. Lüders (Stuttgart, $1975 \mathrm{ff}$.)

B. Gajek: Homo poeta (Frankfurt, 1971)

Jahrbuch, Jahrbücher

Jahrbuch des Freien Deutschen Hochstifts

Jahrbuch der Deutschen Schillergesellschaft 


$\begin{array}{ll}\text { JbSK } & \text { Jahrbuch der Sammlung Kippenberg } \\ \text { JEGPh } & \text { Journal of English and Germanic Philology } \\ \text { JIG } & \text { Jahrbuch für Internationale Germanistik } \\ \text { Kemp } & \text { CB. Werke, hrg. v. Frühwald/Gajek/Kemp, 4 Bde. } \\ & \text { (München, 1968) } \\ \text { LB } & \text { Lujo Brentano: CBs Liebesleben (Frankfurt, 1921) } \\ \text { Linder } & \text { CB. Briefe an Emilie Linder, hrg. v. W. Frühwald (Bad } \\ & \text { Homburg, 1969) } \\ \text { LJb } & \text { Literaturwissenschaftliches Jahrbuch im Auftrage der } \\ & \text { Görres-Gesellschaft } \\ \text { LLZ } & \text { Lieb, Leid und Zeit. Über CB, hrg. v. W. Böhme } \\ & \text { (Karlsruhe, 1979) } \\ \text { MLN } & \text { Modern Language Notes } \\ \text { MLQ } & \text { Modern Language Quarterly } \\ \text { MLR } & \text { Modern Language Review } \\ \text { MR } & \text { CB und Minna Reichenbach. Ungedruckte Briefe des } \\ & \text { Dichters, hrg. v. W. Limburger (Leipzig, 1921) } \\ \text { PMLA } & \text { Publications of the Modern Language Association } \\ \text { Spätwerk } & \text { W. Frühwald: Das Spätwerk CBs (Tübingen, 1977) } \\ \text { Steig } & \text { Achim von Arnim und die ihm nahe standen, hrg. v. } \\ & \text { R. Steig, 3 Bde (Stuttgart, 1894-1913) } \\ \text { Steinle } & \text { Eduard v. Steinle's Briefwechsel mit seinen Freunden, } \\ & \text { hrg. v. A. M. v. Steinle, 2 Bde (Freiburg, 1897) } \\ \text { UL } & \text { Das unsterbliche Leben. Unbekannte Briefe von CB, } \\ \text { hrg. v. W. Schellberg und F. Fuchs (Jena, 1939) } \\ \text { WW } & \text { Wirkendes Wort } \\ \text { ZsfdPh } & \text { Zeitschrift für deutsche Philologie }\end{array}$

